\title{
Effect of the timing of hydrogel spacer placement on prostate and rectal dosimetry of low-dose-rate brachytherapy implants
}

\author{
Wayne M. Butler, PhD, Brian S. Kurko, MS, Whitney J. Scholl, BS, Gregory S. Merrick, MD \\ Schiffler Cancer Center, Wheeling Hospital, Wheeling, WV, USA
}

\begin{abstract}
Purpose: To verify the dose sparing effect of hydrogel spacer (SpaceOAR ${ }^{\mathrm{TM}}$ ) on rectal dosimetry for prostate brachytherapy, and to determine whether prostate and rectal dosimetry was affected by the time gap between hydrogel spacer injection and brachytherapy dosimetry.

Material and methods: The ${ }^{103} \mathrm{Pd}$ brachytherapy dosimetry of 174 consecutive intermediate- and high-risk patients injected with hydrogel was compared with a dosimetry of 174 contemporaneous patients without hydrogel injections. Of the SpaceOAR ${ }^{\mathrm{TM}}$ patients, 91 had hydrogel injected upon completion of brachytherapy implant, while the remaining 83 patients had hydrogel placed prior to external beam radiation therapy (EBRT), followed 2-10 weeks later by brachytherapy. Brachytherapy implants were either planned with the prostate undistorted by any hydrogel or planned with hydrogel in place. Dosimetry of the prostate and tissues at risk was determined from CT imaging on the day of brachytherapy implant.

Results: SpaceOAR ${ }^{\mathrm{TM}}$ significantly reduced mean and maximum rectal doses as well as rectal wall $\mathrm{V}_{50}$, but there was a statistically significant reduction of planning target volume (PTV) $\mathrm{D}_{90}$ to $121.1 \%$ of the prescribed dose in hydrogel patients compared to $123.3 \%$ in the non-hydrogel patients. Rectal dosimetry was similar between patients injected with hydrogel after brachytherapy and those with spacer injected prior to EBRT. However, patients who had hydrogel placed prior to EBRT had statistically significantly higher dosimetry indices of PTV and urethra relative to those with spacer placed at the completion of brachytherapy.

Conclusions: There was a significant rectal dose sparing in the cohort with hydrogel spacer compared to a reference group without spacer injection. The rectal dose sparing effect was similar in the sub-group of patients injected
\end{abstract} with hydrogel prior to EBRT and the sub-group injected with hydrogel at the conclusion of brachytherapy.

Key words: hydrogel spacer, prostate cancer, brachytherapy, rectal dosimetry.

\section{Purpose}

Rectal injury sometimes occurs after radical prostate cancer treatments, such as prostatectomy, external beam radiation therapy (EBRT), and brachytherapy, with a recto-urethral fistula as a rare but profoundly life-altering sequela [1]. In radiation therapy, the dose to the anterior rectum is often a limiting factor, but that dose may be reduced by increasing the separation between the prostate and rectum, either by the presence of perirectal fat or by the injection of some spacer material. Hyaluronic acid injections to decrease radiation-induced rectal toxicity were first reported by Prada et al. in patients undergoing combined modality external beam and high-dose-rate (HDR) brachytherapy [2]. A subsequent clinical trial of a commercial product, the SpaceOAR ${ }^{\mathrm{TM}}$ System (Boston Scientific, Marlborough, MA, USA), confirmed signifi- cant and durable improvements in bowel quality of life (QoL), with lesser improvements in urinary and sexual QoL after use of hydrogel spacer during intensity-modulated radiation therapy (IMRT) [3, 4]. Rectal dose reduction caused by increasing the rectum-prostate separation is also important in salvage brachytherapy of previously irradiated patients $[5,6]$.

After permanent seed, low-dose-rate (LDR) brachytherapy implants, hydrogel spacer insertion has been shown to significantly reduce the most crucial rectal dosimetry parameters and to have minimal effect on target volume dosimetry [7]. The spacer material is usually injected before beginning EBRT or after completion of brachytherapy implant. Higher risk patients have better biochemical survival outcomes if they are treated with combined modalities of external beam radiation thera- 
py and brachytherapy [8]. However, it remains unclear whether hydrogel injection is equally effective when it precedes a course of moderate external beam radiation followed by brachytherapy, or when hydrogel is placed immediately after completion of brachytherapy implant. Asymmetry in hydrogel distribution was found to adversely affect rectal dosimetry in prostate IMRT, and it is expected that displacement of the relatively flat posterior border of the prostate to the one that may be tilted superiorly or inferiorly and/or left vs. right, would adversely affect brachytherapy dosimetry $[9,10]$. An asymmetrical SpaceOAR ${ }^{\mathrm{TM}}$ placement prior to the brachytherapy procedure makes treatment planning and placement of brachytherapy seed implants more difficult and complex due to distortion of the relatively flat posterior prostate. Even with an ideal, symmetric placement of hydrogel, increasing the prostate-rectum separation by about $5 \mathrm{~mm}$ makes execution of a template-guided seed implant more difficult, because more of the anterior prostate may be obstructed by pubic arch interference.

Increasing the time gap between hydrogel spacer placement and brachytherapy implant also increases the amount of hydrogel resorbed. MRI imaging at 12 weeks post-SpaceOAR showed only modest absorption based on differences in rectum-prostate separation compared to the initial separation, but patients imaged just one week later presented with markedly reduced separation [11]. Although the time gap to brachytherapy should not exceed 12 weeks, post-implant rectal dosimetry may be a sensitive indicator of relative loss of rectum-prostate separation. That separation will continue to decrease, as the brachytherapy radionuclide decays - half the total prescribed brachytherapy dose is delivered in one halflife after the implant, and $90 \%$ of the prescribed dose is delivered in 56 days for ${ }^{103} \mathrm{Pd}$ ( 200 days for $\left.{ }^{125} \mathrm{I}\right)$.

This work retrospectively compared the brachytherapy dosimetry of 348 patients, 174 consecutive patients injected with hydrogel SpaceOAR ${ }^{\mathrm{TM}}$ vs. a reference cohort of 174 contemporaneous patients without hydrogel injection. In addition, the dosimetry of two SpaceOAR ${ }^{\mathrm{TM}}$ sub-groups were compared, including 83 patients who had the hydrogel applied before EBRT and brachytherapy vs. 91 patients who had the hydrogel placed at the conclusion of brachytherapy implant.

\section{Material and methods}

The study population consisted of 348 consecutive prostate patients who received a low-dose-rate permanent seed brachytherapy implant between November 2016 and July 2020. This population was divided into two cohorts: 174 men who were injected with SpaceOAR ${ }^{\mathrm{TM}}$ and received brachytherapy between January 2019 and July 2020, and 174 patients not injected with hydrogel who had brachytherapy implants after November 2016. Ninety one of 174 SpaceOAR patients had the hydrogel placed immediately after the brachytherapy implant, and the remaining 83 patients had the hydrogel injected in $57.9 \pm 16.1$ days (mean \pm standard deviation) and median $=63$ days (interquartile range, 50-69 days) prior to brachytherapy implant. In that pre-brachytherapy time period, these patients received a risk-group-specific moderate dose of EBRT as either 2.0 Gy fractions delivering a total of $20 \mathrm{~Gy}$, or $1.8 \mathrm{~Gy}$ fractions delivering either $45 \mathrm{~Gy}$ or $50.4 \mathrm{~Gy}$.

The SpaceOAR ${ }^{\mathrm{TM}}$ hydrogel was injected transperineally under ultrasound guidance after hydro-dissection of Denonvilliers' fascia with sterile saline, following the approach of Pinkawa et al. and the manufacturer's recommendations [12]. For patients prescribed a moderate dose of EBRT, the hydrogel was injected concurrently with implantation of three Varian Calypso ${ }^{\circledR}$ radiofrequency tracking beacons (Varian Medical Systems, Palo Alto, CA, USA) about one week prior to EBRT treatment planning [13]. All the 348 patients in the study were planned and implanted with ${ }^{103} \mathrm{Pd}$ seeds, and had post-implant imaging and dosimetry performed within 24 hours according to the American College of Radiology practice guidelines [14]. Between the SpaceOAR ${ }^{\mathrm{TM}}$ and non-SpaceOAR ${ }^{\mathrm{TM}}$ patients (Table 1), there was no significant difference between the planning or evaluation target volumes, or the number of seeds implanted. The SpaceOAR ${ }^{\mathrm{TM}}$ patients had a statistically higher Gleason score, which, according to our protocols, led to a greater prescribed EBRT dose and a lower brachytherapy prescribed dose (Table 2). Our chosen seed strength was proportional to the brachytherapy prescribed dose and to a lesser extent to the prostate volume. Therefore, the lower brachytherapy prescribed dose for SpaceOAR ${ }^{\mathrm{TM}}$ patients led to a lower mean individual seed strength.

\section{Results}

At post-implant dosimetry, the rectal mean dose, rectal maximum dose, and rectal wall $\mathrm{V}_{50}$ were all statistically significantly lower in the SpaceOAR ${ }^{\mathrm{TM}}$ patients (Table 1). The minimum dose covering $90 \%$ of the planned target volume (PTV D $\mathrm{D}_{90}$ ) was also statistically significantly lower in the SpaceOAR ${ }^{\mathrm{TM}}$ patients, $121.1 \% \pm 10.0 \%$ vs. $123.4 \% \pm 8.7 \%$ in the patients with no hydrogel spacer. The clinical effect of this small dosimetric difference was unclear, and all patients, with or without SpaceOAR ${ }^{\mathrm{TM}}$, met our criteria for acceptable dosimetry, namely, $\mathrm{D}_{90}>100 \%$ of the prescribed dose and $\mathrm{V}_{100}>90 \%$ of the target volume. These data are summarized graphically in Figure 1, and the stark differences in rectal max. dosimetry are illustrated in the dose distribution histograms of Figure 2.

Table 3 compares the sub-groups of SpaceOAR ${ }^{\mathrm{TM}}$ patients, i.e., those injected with the hydrogel before external beam and brachytherapy with those injected with the hydrogel just after the brachytherapy implant. There was no significant difference in rectal dosimetry parameters between the sub-groups. However, in the patients injected with SpaceOAR ${ }^{\mathrm{TM}}$ at the completion of brachytherapy, PTV parameters $\mathrm{D}_{90}$ and $\mathrm{Vpd} \%$ as well as urethral dosimetry parameters were statistically significantly lower than in those patients injected with SpaceOAR ${ }^{\mathrm{TM}}$ prior to any radiation therapy. The bar graphs of Figure 3 place these dosimetric differences in a context: the differences are statistically significant, but their clinical significance is uncertain. 
Table 1. SpaceOAR vs. no SpaceOAR, continuous variables

\begin{tabular}{|c|c|c|c|c|c|c|c|c|c|}
\hline \multirow[t]{2}{*}{ Continuous variable } & \multicolumn{3}{|c|}{ No SpaceOAR $(n=174)$} & \multicolumn{3}{|c|}{ SpaceOAR $(n=174)$} & \multirow[t]{2}{*}{$p$-value ${ }^{\dagger}$} & \multicolumn{2}{|c|}{ Total pts. $(N=348)$} \\
\hline & Mean & SD & Median & Mean & SD & Median & & Mean & SD \\
\hline Age at implant (years) & 64.8 & $(6.7)$ & 64.3 & 64.8 & $(7.4)$ & 65.2 & 0.914 & 64.8 & (7.0) \\
\hline Pre-Tx PSA (ng/ml) & 10.6 & $(13.9)$ & 6.7 & 9.5 & (8.9) & 6.8 & 0.367 & 10.1 & (11.6) \\
\hline Gleason score & 7.3 & $(0.6)$ & 7.0 & 7.5 & $(0.8)$ & 7.0 & 0.005 & 7.4 & $(0.7)$ \\
\hline TRUS prostate volume $\left(\mathrm{cm}^{3}\right)$ & 23.4 & $(6.1)$ & 23.4 & 23.1 & $(6.0)$ & 23.2 & 0.625 & 23.3 & $(6.0)$ \\
\hline Planning target volume, PTV $\left(\mathrm{cm}^{3}\right)$ & 48.0 & (9.8) & 47.3 & 48.2 & $(10.0)$ & 48.4 & 0.834 & 48.1 & (9.9) \\
\hline Post-implant CT volume $\left(\mathrm{cm}^{3}\right)$ & 64.3 & $(12.3)$ & 63.2 & 65.9 & $(11.9)$ & 64.9 & 0.221 & 65.1 & $(12.1)$ \\
\hline Plan vol. of Rx isodose $\left(\mathrm{cm}^{3}\right)$ & 85.3 & (15.7) & 83.7 & 87.7 & $(15.8)$ & 87.3 & 0.159 & 86.5 & (15.8) \\
\hline CT vol. of Rx isodose $\left(\mathrm{cm}^{3}\right)$ & 97.8 & (6.9) & 98.2 & 99.4 & $(14.8)$ & 99.0 & 0.216 & 98.6 & (11.5) \\
\hline Number of seeds & 104.3 & $(11.3)$ & 103.0 & 106.0 & $(11.6)$ & 105.5 & 0.185 & 105.2 & $(11.5)$ \\
\hline Seed strength $(U)$ & 2.55 & $(0.48)$ & 2.32 & 2.41 & $(0.40)$ & 2.23 & 0.004 & 2.48 & $(0.45)$ \\
\hline Body mass index $\left(\mathrm{kg} / \mathrm{m}^{2}\right)$ & 30.0 & $(5.5)$ & 29.3 & 30.5 & $(6.0)$ & 29.6 & 0.372 & 30.2 & $(5.8)$ \\
\hline PTV $D_{90}(\%)$ & 123.4 & $(8.7)$ & 122.6 & 121.1 & $(10.0)$ & 119.9 & 0.025 & 122.2 & $(9.4)$ \\
\hline PTV $V_{100}(\%)$ & 97.9 & $(1.5)$ & 98.3 & 97.7 & $(1.8)$ & 98.3 & 0.254 & 97.8 & $(1.6)$ \\
\hline PTV $V_{150}(\%)$ & 73.3 & (6.6) & 73.0 & 72.0 & $(7.3)$ & 72.0 & 0.070 & 72.7 & (7.0) \\
\hline PTV V $200(\%)$ & 44.9 & $(6.1)$ & 44.3 & 44.6 & (6.9) & 43.6 & 0.671 & 44.7 & $(6.5)$ \\
\hline Prostatic urethral dose (\%) & 112.9 & $(12.2)$ & 111.9 & 111.8 & $(12.0)$ & 109.8 & 0.397 & 112.4 & $(12.1)$ \\
\hline Prostatic urethral dose, max. (\%) & 130.5 & $(17.2)$ & 129.5 & 127.2 & $(19.0)$ & 123.2 & 0.096 & 128.9 & $(18.2)$ \\
\hline Bulbomembranous dose (Gy) & 33.0 & (12.4) & 31.4 & 34.6 & $(14.3)$ & 31.2 & 0.288 & 33.8 & (13.4) \\
\hline Rectal dose (\%) & 34.2 & (15.4) & 32.3 & 22.8 & $(8.5)$ & 20.8 & $<0.001$ & 28.5 & $(13.7)$ \\
\hline Rectal dose, max. (\%) & 51.8 & $(23.0)$ & 47.9 & 32.6 & $(12.0)$ & 30.4 & $<0.001$ & 42.2 & $(20.7)$ \\
\hline Rectum $V_{110}\left(\mathrm{~cm}^{3}\right)$ & 0.009 & $(0.033)$ & 0.0 & 0.008 & $(0.086)$ & 0.0 & 0.869 & 0.009 & $(0.065)$ \\
\hline Rectal wall $V_{50}\left(\mathrm{~cm}^{3}\right)$ & 1.90 & $(1.07)$ & 1.88 & 0.58 & $(0.77)$ & 0.21 & $<0.001$ & 1.90 & $(1.07)$ \\
\hline
\end{tabular}

${ }^{\dagger}$ Two sample t-test, $p$-values $\leq 0.5$ in bold, PTV - planned target volume, $D_{90}$ - minimum dose to the hottest $90 \%$ of the volume as percentage of the prescribed (Rx) dose, $\mathrm{Vpd}$-volume receiving pd percent of the prescribed PTV dose as percentage of the structure volume or $\mathrm{cm}^{3}$, as specified

Table 2. SpaceOAR vs. no SpaceOAR, categorical variables

\begin{tabular}{|c|c|c|c|c|c|c|c|c|}
\hline \multirow[t]{2}{*}{ Categorical variable } & & \multicolumn{2}{|c|}{ No SpaceOAR } & \multicolumn{2}{|c|}{ SpaceOAR } & \multicolumn{2}{|c|}{ Total } & \multirow[t]{2}{*}{$p$-value } \\
\hline & & $n$ & $\%$ & $n$ & $\%$ & $n$ & $\%$ & \\
\hline \multirow[t]{5}{*}{ Clinical stage } & T1c & 134 & 77.0 & 123 & 70.7 & 257 & 73.8 & \multirow[t]{5}{*}{0.349} \\
\hline & $\mathrm{T} 2 \mathrm{a}$ & 26 & 14.9 & 34 & 19.5 & 60 & 17.2 & \\
\hline & $\mathrm{T} 2 \mathrm{~b}$ & 9 & 5.2 & 8 & 4.6 & 17 & 4.9 & \\
\hline & $\mathrm{T} 2 \mathrm{C}$ & 5 & 2.9 & 4 & 2.3 & 9 & 2.6 & \\
\hline & $T 3 a, b, c$ & 0 & 0.0 & 5 & 2.9 & 5 & 1.4 & \\
\hline \multirow{4}{*}{$\begin{array}{l}{ }^{103} \mathrm{Pd} \text { implant Rx dose } \\
\text { (Gy) }\end{array}$} & 80 & 90 & 51.7 & 106 & 60.9 & 196 & 56.3 & \multirow[t]{4}{*}{0.005} \\
\hline & 90 & 22 & 12.6 & 34 & 19.5 & 56 & 16.1 & \\
\hline & 115 & 29 & 16.7 & 22 & 12.6 & 51 & 14.7 & \\
\hline & 125 & 33 & 19.0 & 12 & 16.9 & 45 & 12.9 & \\
\hline \multirow[t]{4}{*}{ NCCN Risk Group } & Low & 3 & 1.7 & 0 & 0.0 & 3 & 0.9 & \multirow[t]{4}{*}{0.061} \\
\hline & Intermediate & 119 & 68.4 & 113 & 64.9 & 232 & 66.7 & \\
\hline & High & 52 & 29.9 & 57 & 32.8 & 109 & 31.3 & \\
\hline & Very high & 0 & 0.0 & 4 & 2.3 & 4 & 1.2 & \\
\hline \multirow[t]{2}{*}{ Diabetes } & No & 173 & 99.4 & 174 & 100.0 & 347 & 99.7 & \multirow[t]{2}{*}{0.317} \\
\hline & Yes & 1 & 0.6 & 0 & 0.0 & 1 & 0.3 & \\
\hline \multirow[t]{2}{*}{ Hypercholesterolemia } & No & 172 & 98.8 & 174 & 100.0 & 346 & 99.4 & \multirow[t]{2}{*}{0.156} \\
\hline & Yes & 2 & 1.2 & 0 & 0.0 & 2 & 0.6 & \\
\hline \multirow[t]{2}{*}{ Hypertension } & No & 171 & 98.3 & 174 & 100.0 & 345 & 99.1 & \multirow[t]{2}{*}{0.082} \\
\hline & Yes & 3 & 1.7 & 0 & 0.0 & 3 & 0.9 & \\
\hline
\end{tabular}

Chi-squared test, $p$-values $\leq 0.5$ in bold 


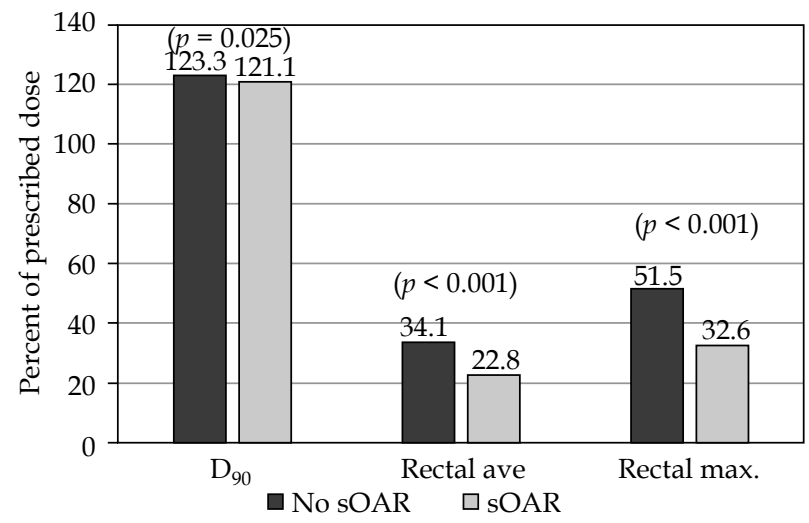

Fig. 1. Mean dosimetric parameters of patients implanted with SpaceOAR hydrogel (sOAR, $n=174$ ) and a reference cohort of patients with no SpaceOAR (no sOAR, $n=174$ ). The dosimetry difference between the two patient groups was statistically significant $(p \leq 0.05)$ for prostate target volume $\mathrm{D}_{90}$, rectal average dose, and rectal maximum dose

It is noteworthy that in the sub-group injected with SpaceOAR ${ }^{\mathrm{TM}}$ prior to any radiation therapy, the $\mathrm{PTV}_{90}$ and $V_{100}$ means were nearly identical with the reference cohort not injected with the hydrogel. Table 4 details the differences between the non-hydrogel reference group and the two SpaceOAR ${ }^{\mathrm{TM}}$ sub-groups. PTV and urethral dosimetry parameters of the sub-group receiving Space$\mathrm{OAR}^{\mathrm{TM}}$ prior to EBRT were not significantly different from the reference cohort. However, the sub-group receiving SpaceOAR at the time of brachytherapy had statistically significantly lower mean PTV and urethral dosimetry than those patients not receiving any hydrogel. Both the SpaceOAR ${ }^{\mathrm{TM}}$ sub-groups (prior to and after brachytherapy) had significantly lower mean and

A

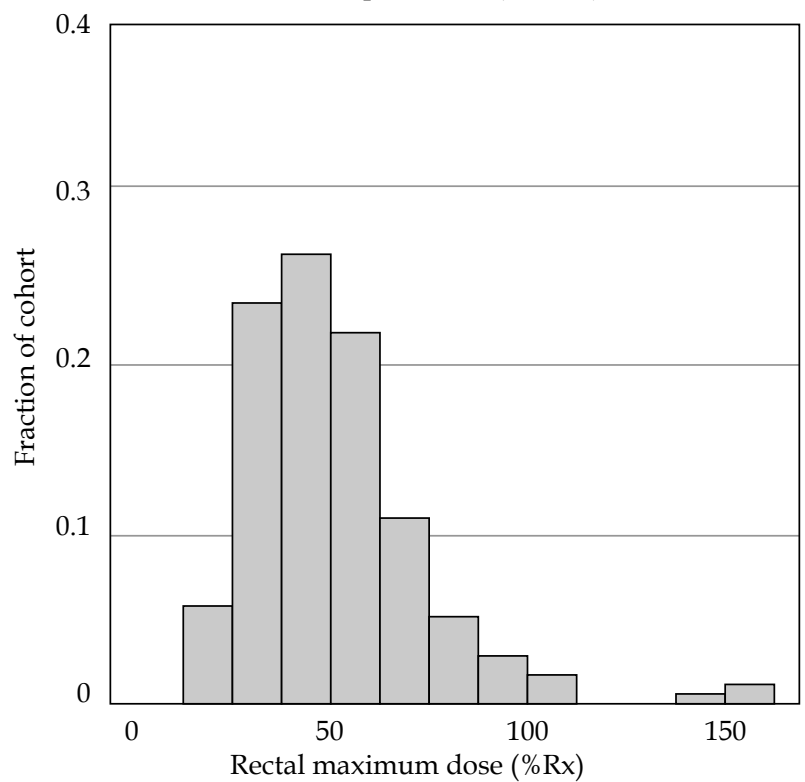

maximum rectal doses than the non-hydrogel reference cohort.

Because the injected hydrogel was not always a uniform $5 \mathrm{~mm}$ spacer between the prostate and rectum, treatment plans sometimes placed seeds within the gel rather than within the prostate tissue. Our protocols require expansion of the prostate by about $5 \mathrm{~mm}$ in all directions except posteriorly to create the PTV, which has a planning goal of $\mathrm{V}_{100}>99.9 \%$. This has always entailed a large number of seeds outside the prostate and some outside the PTV. However, planned placement of seeds posterior to the PTV is an unusual feature of some SpaceOAR ${ }^{\mathrm{TM}}$ EBRT patients. Adding about $5 \mathrm{~mm}$ of rectal spacing necessarily raises the prostate, thus increasing the likelihood of pubic arch interference. This was addressed by adjusting the patient's leg position to open the pelvis or to insert the implant needles on an upward trajectory to get behind the pubic arch. This occurred in the SpaceOAR ${ }^{\mathrm{TM}}$ and non-SpaceOAR ${ }^{\mathrm{TM}}$ cohorts, and the intra-operative dosimetry was unremarkable. Since the seed placement produced satisfactory dosimetry in all cases, it was not noteworthy, and therefore our documentation was insufficient to determine if arch interference was more common in EBRT sub-groups.

\section{Discussion}

For LDR prostate brachytherapy treatments, the dose to the rectum in those patients with injected spacer material is considerably less than in non-spacer control groups. This is consistent with the results reported by Kahn et al. [15] for ${ }^{125}$ I brachytherapy and by Taggar et al. [7] for ${ }^{103} \mathrm{Pd}$ brachytherapy. We did find one rectal dose parameter that was not significantly different between the SpaceOAR ${ }^{\mathrm{TM}}$ and non-SpaceOAR ${ }^{\mathrm{TM}}$ cohorts: the rectal

B Space OAR $(n=174)$

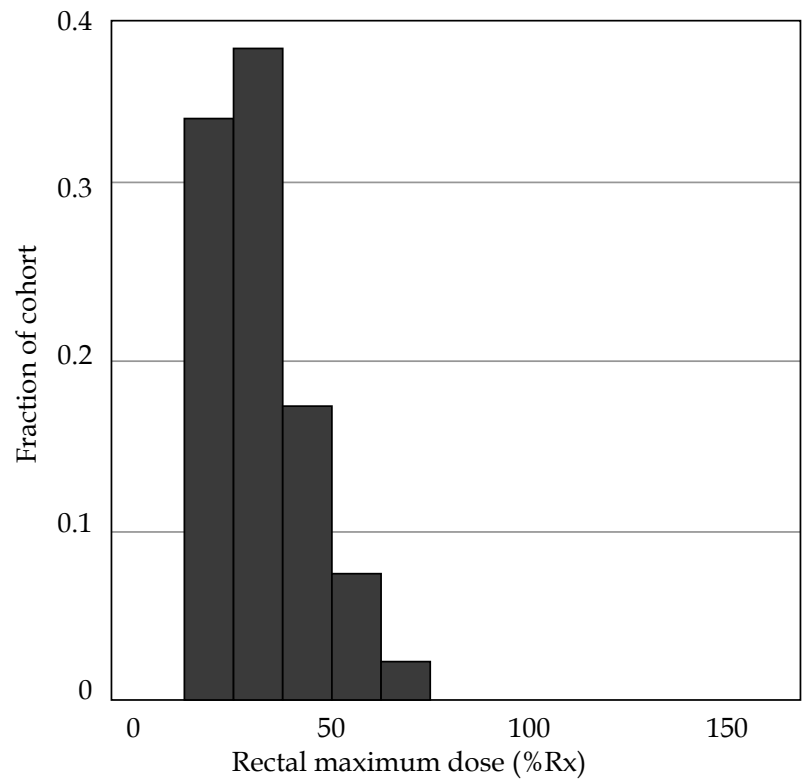

Fig. 2. Histograms of rectal maximum dose as a percentage of the prescribed dose (\%Rx) for the two equal-sized cohorts: patients without SpaceOAR (A, reference) and patients implanted with SpaceOAR hydrogel (B). The latter group is more tightly skewed toward low doses 
Table 3. Patients who received SpaceOAR prior to any radiation therapy compared to those who received SpaceOAR on the day of brachytherapy seed implant

\begin{tabular}{|c|c|c|c|c|c|c|c|}
\hline \multirow[t]{2}{*}{ Continuous variable } & \multicolumn{2}{|c|}{$\begin{array}{c}\text { SpaceOAR before XRT } \\
(n=83)\end{array}$} & \multicolumn{2}{|c|}{$\begin{array}{l}\text { SpaceOAR at brachy } \\
(n=91)\end{array}$} & \multirow[t]{2}{*}{$p$-value ${ }^{\dagger}$} & \multicolumn{2}{|c|}{$\begin{array}{c}\text { Total SpaceOAR pts } \\
\qquad(n=174)\end{array}$} \\
\hline & Mean & SD & Mean & SD & & Mean & SD \\
\hline TRUS prostate volume $\left(\mathrm{cm}^{3}\right)$ & 22.7 & $(6.3)$ & 23.5 & $(5.7)$ & 0.366 & 23.1 & $(6.0)$ \\
\hline Planning target volume, PTV $\left(\mathrm{cm}^{3}\right)$ & 48.0 & (10.5) & 48.3 & (9.6) & 0.828 & 48.2 & $(10.0)$ \\
\hline Post-implant CT volume $\left(\mathrm{cm}^{3}\right)$ & 65.1 & $(12.6)$ & 66.7 & $(11.3)$ & 0.389 & 65.9 & $(11.9)$ \\
\hline PTV $D_{90}(\%)$ & 123.3 & $(10.7)$ & 119.1 & $(9.0)$ & 0.005 & 121.1 & $(10.0)$ \\
\hline PTV V $100(\%)$ & 98.0 & $(1.6)$ & 97.4 & $(1.9)$ & 0.024 & 97.7 & $(1.8)$ \\
\hline PTV V $V_{150}(\%)$ & 73.4 & $(7.1)$ & 70.7 & $(7.2)$ & 0.012 & 72.0 & $(7.3)$ \\
\hline PTV V $200(\%)$ & 46.1 & $(7.1)$ & 43.2 & $(6.5)$ & 0.005 & 44.6 & $(6.9)$ \\
\hline Prostatic urethral dose (\%) & 115.4 & $(14.1)$ & 108.5 & $(8.5)$ & $<0.001$ & 111.8 & $(12.0)$ \\
\hline Prostatic urethral dose, max. (\%) & 132.8 & $(22.1)$ & 122.2 & $(14.0)$ & $<0.001$ & 127.2 & $(19.0)$ \\
\hline Bulbomembranous dose (Gy) & 34.9 & $(12.7)$ & 34.3 & $(15.8)$ & 0.772 & 34.6 & $(14.3)$ \\
\hline Rectal dose (\%) & 23.8 & $(8.5)$ & 21.8 & $(8.5)$ & 0.122 & 22.8 & $(8.5)$ \\
\hline Rectal dose, max. (\%) & 33.9 & $(12.5)$ & 31.4 & $(11.5)$ & 0.173 & 32.6 & $(12.0)$ \\
\hline Rectum $\vee_{110}\left(\mathrm{~cm}^{3}\right)$ & 0.002 & $(0.016)$ & 0.014 & $(0.118)$ & 0.349 & 0.008 & $(0.086)$ \\
\hline Rectal wall $V_{50}\left(\mathrm{~cm}^{3}\right)$ & 0.596 & $(0.801)$ & 0.571 & $(0.754)$ & 0.840 & 0.583 & $(0.775)$ \\
\hline
\end{tabular}

${ }^{\dagger}$ Two sample t-test, $p$-values $\leq 0.5$ in bold

$\mathrm{V}_{110}$. Because of the rapid dose fall-off with distance for ${ }^{103} \mathrm{Pd}$ seeds, the rectal volume receiving $110 \%$ of the prescribed prostate dose approached zero, and the median for both cohorts was zero $\mathrm{cm}^{3}$.

In their ${ }^{125} \mathrm{I}$ brachytherapy study, Kahn and colleagues found no significant difference between prostate dosimetry parameters in patients with or without hydrogel spacer, but the ${ }^{103} \mathrm{Pd}$ brachytherapy study of Taggar and colleagues did find a significantly higher prostate $\mathrm{V}_{150}$ and $\mathrm{V}_{200}$ in hydrogel group. This is contrary to our finding of a significantly lower $\mathrm{D}_{90}$ in the hydrogel group and no significant difference in the other PTV indices. All patients in the Taggar study had the hydrogel injected at the completion of brachytherapy implant; therefore, the proper comparison group in our study was the Space$\mathrm{OAR}^{\mathrm{TM}}$ at brachytherapy sub-group. The patients in this sub-group relative to the non-hydrogel cohort had statistically significantly cooler PTV $\mathrm{D}_{90}, \mathrm{~V}_{100}, \mathrm{~V}_{150}, \mathrm{~V}_{200}$, and the prostatic urethra dosimetry parameters. Some of the difference between our target volume and urethral dosimetry and that of the Taggar group may be due to implant approach. Creating a PTV by adding 5 to $7 \mathrm{~mm}$ to the prostate makes the PTV about twice the volume of the prostate. Our PTV V 100 goal of $>99 \%$ resulted in placing a large fraction of the seeds outside the prostate, and only a small number interior to the prostate and very few within $10 \mathrm{~mm}$ of the urethra.

The difference between the SpaceOAR ${ }^{\mathrm{TM}}$ sub-groups in terms of PTV and urethral dosimetry may be multifactorial. The patients injected with hydrogel at the completion of brachytherapy experience not only prostatic edema from implant trauma and radiation, but also distortion from the freshly placed hydrogel and edema due to infiltration into the prostate of the saline solution, creating the initial space for the hydrogel. In this work, we did not measure the dimensions of the prostate post-implant, so the hypothesis of distortion and additional edema caused by the hydrogel must await a future study.

In higher risk patients, we have always performed EBRT first followed by brachytherapy implant within one week of EBRT completion. The reverse sequence requires a time gap of one to two half-lives of the radionuclide to reduce the overlay of brachytherapy and EBRT doses. This gap adds to the radiobiological uncertainty of the total biological effective dose (BED) due to poorly charac-

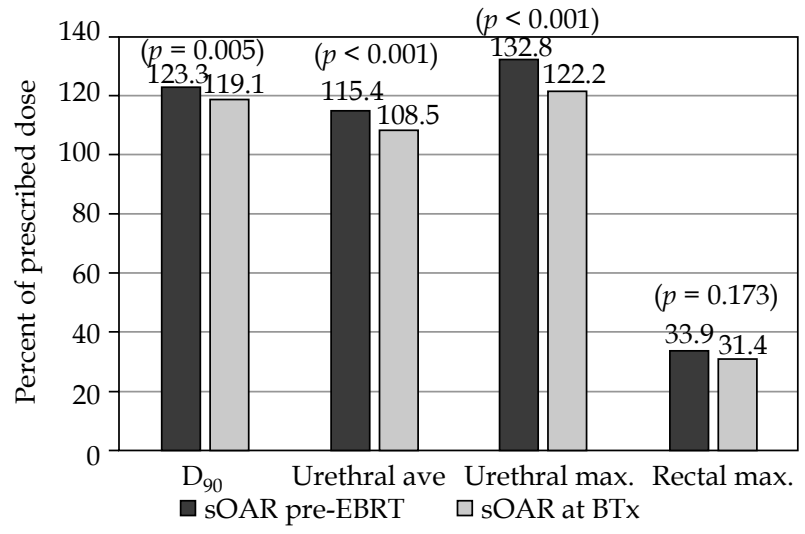

Fig. 3. Mean dosimetric parameters of SpaceOAR subgroups: patients implanted with SpaceOAR hydrogel before any radiation therapy (sOAR pre-EBRT, $n=83$ ) and patients implanted with SpaceOAR hydrogel after completion of a brachytherapy implant (sOAR at BTx, $n=91$ ). The dosimetry difference between the two patient groups was statistically significant $(p \leq 0.05)$ for the prostate target volume $\mathrm{D}_{90}$, urethral average dose, and urethral maximum dose, but they were not significantly different for any rectal dosimetry parameters 
Table 4. Patients who received no SpaceOAR (reference group) compared to those who received SpaceOAR prior to any radiation therapy and compared to those who received SpaceOAR on the day of brachytherapy seed implant

\begin{tabular}{|c|c|c|c|c|c|c|c|c|}
\hline \multirow[t]{2}{*}{ Continuous variable } & \multicolumn{2}{|c|}{$\begin{array}{c}\text { No SpaceOAR } \\
\quad(n=174)\end{array}$} & \multicolumn{2}{|c|}{$\begin{array}{c}\text { SpaceOAR before } \\
\text { XRT } \\
(n=83) \\
\end{array}$} & \multicolumn{2}{|c|}{$\begin{array}{l}\text { SpaceOAR at brachy } \\
\qquad(n=91)\end{array}$} & \multirow[t]{2}{*}{$p$-value ${ }^{\dagger}$} & \multirow[t]{2}{*}{$p$-value ${ }^{\ddagger}$} \\
\hline & Mean & SD & Mean & SD & Mean & SD & & \\
\hline TRUS prostate volume $\left(\mathrm{cm}^{3}\right)$ & 23.4 & $(6.1)$ & 22.7 & $(6.3)$ & 23.5 & $(5.7)$ & 0.362 & 0.919 \\
\hline Planning target volume, PTV $\left(\mathrm{cm}^{3}\right)$ & 48.0 & (9.8) & 48.0 & $(10.5)$ & 48.3 & (9.6) & 0.970 & 0.763 \\
\hline PTV $D_{90}(\%)$ & 123.4 & $(8.7)$ & 123.3 & $(10.7)$ & 119.1 & (9.0) & 0.973 & $<0.001$ \\
\hline PTV $V_{100}(\%)$ & 97.9 & $(1.5)$ & 98.0 & $(1.6)$ & 97.4 & $(1.9)$ & 0.563 & 0.020 \\
\hline PTV $V_{150}(\%)$ & 73.3 & $(6.6)$ & 73.4 & $(7.1)$ & 70.7 & $(7.2)$ & 0.910 & 0.003 \\
\hline PTV V $200(\%)$ & 44.9 & $(6.1)$ & 46.1 & $(7.1)$ & 43.2 & $(6.5)$ & 0.148 & 0.036 \\
\hline Prostatic urethral dose (\%) & 112.9 & $(12.2)$ & 115.4 & $(14.1)$ & 108.5 & $(8.5)$ & 0.141 & 0.002 \\
\hline Prostatic urethral dose, max. (\%) & 130.5 & $(17.2)$ & 132.8 & $(22.1)$ & 122.2 & $(14.0)$ & 0.359 & $<0.001$ \\
\hline Bulbomembranous dose (Gy) & 33.0 & $(12.4)$ & 34.9 & $(12.7)$ & 34.3 & $(15.8)$ & 0.264 & 0.492 \\
\hline Rectal dose (\%) & 34.2 & $(15.4)$ & 23.8 & $(8.5)$ & 21.8 & $(8.5)$ & $<0.001$ & $<0.001$ \\
\hline Rectal dose, max. (\%) & 51.8 & $(23.0)$ & 33.9 & $(12.5)$ & 31.4 & $(11.5)$ & $<0.001$ & $<0.001$ \\
\hline Rectum $\vee_{110}\left(\mathrm{~cm}^{3}\right)$ & 0.009 & $(0.033)$ & 0.002 & $(0.016)$ & 0.014 & $(0.118)$ & 0.050 & 0.624 \\
\hline Rectal wall $V_{50}\left(\mathrm{~cm}^{3}\right)$ & 1.90 & $(1.07)$ & 0.596 & $(0.801)$ & 0.571 & $(0.754)$ & $<0.001$ & $<0.001$ \\
\hline
\end{tabular}

${ }^{\dagger}$ Two sample t-test comparing No SpaceOAR with SpaceOAR before XRT, p-values $\leq 0.5$ in bold, "two sample t-test comparing No SpaceOAR with SpaceOAR at brachytherapy, $p$-values $\leq 0.5$ in bold

terized repair and repopulation rates. However, this sequence should be considered in the case of patients with marginal or actual pubic arch interference at the initial prostate volume study, because of the increased technical difficulty of implant after the hydrogel spacer, which raises the prostate.

Limitations of this study include its retrospective design. Prostate brachytherapy involves dynamic processes of radioactive decay, the inception and patient variable resolution of edema due to implant trauma and radiation, the resorption of implanted hydrogel, and the possible migration and loss of implanted seeds. Sequential post-implant dosimetry studies as part of a clinical trial would have provided more granular temporal information, but we chose to use day 0 dosimetry, which is a protocol we have used for all our prostate brachytherapy patients. The AAPM Task Group 137 recommends post-implant imaging on either day 0 or at the nominal optimal dosimetry time for the radionuclide being used [16]. Some of our patients on clinical trials had additional imaging studies, but none of those were part of this study. Although some EBRT patients were implanted in the time period when the spacer is being absorbed, the spacer has already served its primary purpose of protecting the rectum from EBRT.

\section{Conclusions}

In a study population treated with LDR prostate brachytherapy, the hydrogel SpaceOAR injected between the prostate and rectal wall to increase the separation between the prostate and rectum, significantly reduced rectal dosimetry values compared to the reference group without spacer injection. The rectal dose sparing effect was similar in the sub-group of patients injected with the hydrogel prior to EBRT (2-10 weeks prior to the brachytherapy implant) and the sub-group patients injected with the hydrogel at the conclusion of the brachytherapy implant.

\section{Disclosure}

The authors report no conflict of interest.

\section{References}

1. Harris CR, McAninch JW, Mundy AR et al. Rectourethral fistulas secondary to prostate cancer treatment: management and outcomes from a multi-institutional combined experience. J Urol 2017; 197: 191-194.

2. Prada PJ, Fernandez J, Martinez AA et al. Transperineal injection of hyaluronic acid in anterior perirectal fat to decrease rectal toxicity from radiation delivered with intensity modulated brachytherapy or EBRT for prostate cancer patients. Int I Radiat Oncol Biol Phys 2007; 69: 95-102.

3. Hamstra DA, Mariados N, Sylvester J et al. Continued benefit to rectal separation for prostate radiation therapy: final results of a phase III trial. Int J Radiat Oncol Biol Phys 2017; 97: 976-985

4. Hamstra DA, Mariados N, Sylvester J et al. Sexual quality of life following prostate intensity modulated radiation therapy (IMRT) with a rectal/prostate spacer: Secondary analysis of a phase 3 trial. Pract Radiat Oncol 2018; 8: e7-e15.

5. Mahal BA, Ziehr DR, Hyatt AS et al. Use of a rectal spacer with low-dose-rate brachytherapy for treatment of prostate cancer in previously irradiated patients: Initial experience and short-term results. Brachytherapy 2014; 13: 442-449.

6. Guimas V, Quivrin M, Bertaut A et al. Focal or whole-gland salvage prostate brachytherapy with iodine seeds with or without a rectal spacer for postradiotherapy local failure: 
How best to spare the rectum? Brachytherapy 2016; 15: 406411.

7. Taggar AS, Charas T, Cohen GN et al. Placement of an absorbable rectal hydrogel spacer in patients undergoing lowdose-rate brachytherapy with palladium-103. Brachytherapy 2018; 17: 251-258.

8. Kishan AU, Cook RR, Ciezki JP et al. Radical prostatectomy, external beam radiotherapy, or external beam radiotherapy with brachytherapy boost and disease progression and mortality in patients with Gleason score 9-10 prostate cancer. JAMA 2018; 319: 896-905.

9. Fischer-Valuck BW, Chundury A, Gay H et al. Hydrogel spacer distribution within the perirectal space in patients undergoing radiotherapy for prostate cancer: Impact of spacer symmetry on rectal dose reduction and the clinical consequences of hydrogel infiltration into the rectal wall. Pract Radiat Oncol 2017; 7: 195-202.

10. Liu H, Borden L, Wiant D et al. Proposed hydrogel-implant-quality-score (HIQS) and a matched-pair study for prostate radiotherapy. Pract Radiat Oncol 2020; 10: 202-208.

11. Mariados N, Sylvester J, Shah D et al. Hydrogel spacer prospective multicenter randomized controlled pivotal trial: dosimetric and clinical effects of perirectal spacer application in men undergoing prostate image guided intensity modulated radiation therapy. Int J Radiat Oncol Biol Phys 2015; 92: 971-977.

12. Pinkawa M, Corral NE, Caffaro $M$ et al. Application of a spacer gel to optimize three-dimensional conformal and intensity modulated radiotherapy for prostate cancer. Radiother Oncol 2011; 100: 436-441.

13. Butler WM, Merrick GS, Reed JL et al. Intrafraction displacement of prone versus supine prostate positioning monitored by real-time electromagnetic tracking. J Appl Clin Med Phys 2013; 14: 4141.

14. Bittner NH, Orio PF, 3rd, Merrick GS et al. The American College of Radiology and the American Brachytherapy Society practice parameter for transperineal permanent brachytherapy of prostate cancer. Brachytherapy 2017; 16: 59-67.

15. Kahn J, Dahman B, McLaughlin C et al. Rectal spacing, prostate coverage, and periprocedural outcomes after hydrogel spacer injection during low-dose-rate brachytherapy implantation. Brachytherapy 2020; 19: 228-233.

16. Nath R, Bice WS, Butler WM et al. AAPM recommendations on dose prescription and reporting methods for permanent interstitial brachytherapy for prostate cancer: report of Task Group 137. Med Phys 2009; 36: 5310-5322. 